\title{
Metallothionein Inhibits Myocardial Apoptosis in Copper-Deficient Mice: Role of Atrial Natriuretic Peptide
}

\author{
Y. James Kang, Zhan-Xiang Zhou, Huiyun Wu, Guang-Wu Wang, Jack T. Saari, and \\ Jon B. Klein
}

Departments of Medicine (YJK, Z-XZ, HW, G-WW, JBK), and Pharmacology and Toxicology (YJK), University of Louisville; Jewish Hospital, Heart and Lung Institute (YJK) and Veterans Affairs Medical Center (JBK), Louisville, Kentucky; and the US Department of Agriculture (JTS), Agricultural Research Service, Grand Forks Human Nutrition Research Center, Grand Forks, North Dakota

\begin{abstract}
SUMMARY: Dietary copper restriction causes heart hypertrophy in animal models. Several studies have indicated that this cardiomyopathy is mediated by oxidative stress. Metallothionein (MT), a low molecular weight and cysteine-rich protein, functions in protecting the heart from oxidative injury. We therefore used a cardiac-specific MT-overexpressing transgenic mouse model to test the hypothesis that MT inhibits copper deficiency-induced heart hypertrophy. Dams of both transgenic pups and non-transgenic littermates were fed a copper-adequate or copper-deficient diet, starting on the fourth day post-delivery, and the weanling mice were continued on the dams' diets until they were killed. Heart hypertrophy developed in copper-deficient pups by the fourth week of the combined pre- and post-weaning feeding and aggressively progressed until the end of the experiment (6 weeks). MT overexpression did not prevent the occurrence of heart hypertrophy, but inhibited the progression of this cardiomyopathy, which correlated with its suppression of cardiac lipid peroxidation. Corresponding to the progression of heart hypertrophy, myocardial apoptosis and atrial natriuretic peptide (ANP) production in the left ventricle were detected in non-transgenic copper-deficient mice; these effects were significantly suppressed in transgenic copper-deficient mice. Measurement of apoptosis by TUNEL assay and Annexin V-FITC confocal microscopy in primary cultures of cardiomyocytes revealed that ANP was largely responsible for the myocyte apoptosis and that MT inhibited ANP-induced apoptosis. The data clearly demonstrate that elevation of MT in the heart inhibits oxidative injury and suppresses the progression of heart hypertrophy in copper deficiency, although it does not block its initiation. The results suggest that MT inhibits the transition from heart hypertrophy to failure by suppressing apoptosis through inhibition of both cardiac ANP production and its apoptotic effect. (Lab Invest 2000, 80:745-757).
\end{abstract}

$D$ ietary copper restriction results in retarded growth and impaired reproduction in many species, including humans (Davis and Mertz, 1987). Alterations of morphology, function, and biochemical pathways in many organs of copper-deficient animals have been observed (Fell, 1987; Prohaska, 1990). The heart is often severely affected by copper deficiency, exhib-

Received February 15, 2000.

This research was supported in part by U.S. Department of Agriculture grant \#9604531, National Institutes of Health grants HL59225 and CA68125, American Heart Association Established Investigator Award (9640091N), and Jewish Hospital Foundation, Louisville, Kentucky.

Mention of a trademark or proprietary product does not constitute a guarantee or warranty of the product by the U.S. Department of Agriculture and does not imply its approval to the exclusion of other products that may be suitable.

The U.S. Department of Agriculture, Agricultural Research Service, Northern Plains Area, is an equal opportunity/affirmative action employer and all agency services are available without discrimination.

Address reprint requests to: Dr. Y. James Kang, Department of Medicine, University of Louisville School of Medicine, 511 S. Floyd St., MDR 530, Louisville, KY 40292. Fax: 502-852-6904; E-mail: yjkang01@athena. louisville.edu iting myocyte enlargement and mitochondrial swelling (Medeiros et al, 1991), as well as associated depression in electrical (Viestenz and Klevay, 1982), contractile (Prohaska and Heller, 1982), and respiratory function (Bode et al, 1992), which leads to heart failure (Medeiros et al, 1991).

Most studies on the heart hypertrophy induced by copper deficiency have focused on its cause, while its important consequence, heart failure, has largely been neglected. It is well known that pathological heart hypertrophy will inevitably lead to heart failure. The transition from heart hypertrophy to failure is a very important experimental as well as clinical problem. Current progress in cardiac research suggests that myocardial apoptosis plays a critical role in the transition, as supported by accumulated evidence from both animal and human studies (Lorell, 1997; Sabbah and Sharov, 1998). It is thus important to know whether this cellular event occurs in the copperdeficient heart.

Several studies have focused on the effects of copper deficiency on cuproenzymes in the heart and their role in heart hypertrophy (Medeiros et al, 1993; 
Prohaska, 1990). Three major components have been considered: (1) the antioxidant system, in particular, copper/zinc-dependent superoxide dismutase $(\mathrm{Cu}$, $\mathrm{Zn}-\mathrm{SOD}$ ); (2) connective tissue function and lysyl oxidase; and (3) energy metabolism, as affected by cytochrome-c oxidase (CCO). All three of these enzymes show decreased activities with copper deficiency in experimental animals. Decreased Cu, ZnSOD activity in the heart has been shown to correlate with increased accumulation of reactive oxygen species and lipid peroxidation (Chen et al, 1994). Suppressed lysyl oxidase activity leads to decreased collagen cross-linking, thereby weakening the myocardium and causing an increased work load (Borg et al, 1985). CCO is a copper-dependent electron transport enzyme; the levels of the nuclear-encoded subunits of $\mathrm{CCO}$ are markedly decreased, suggesting altered energy metabolism (Chao et al, 1994). However, the cause-and-effect relationship between the depressed enzyme activities and heart hypertrophy by dietary copper restriction has not been demonstrated.

Besides suppressing the activity of coppercontaining SOD, copper deficiency also decreases non-copper-containing antioxidant enzymes (Prohaska, 1990). This has led to many studies that have pursued the hypothesis that oxidative stress is involved in the pathophysiology of copper deficiency. A substantial amount of support exists for an oxidative mechanism of copper deficiency-induced heart damage. This includes inhibition of cardiac defects by antioxidants (Saari, 1989), increased susceptibility of mitochondria to in vitro oxidation (Akers and Saari, 1993; Bode et al, 1992; Nelson et al, 1992), enhanced tissue damage with oxidative stress (Jenkinson et al, 1984), and increased lipid peroxidation (Balevska et al, 1981 ) in copper-deficient animals. In studies to determine the role of oxy-radicals in copper deficiencyinduced heart hypertrophy, dimethyl sulfoxide (DMSO), an antioxidant, was administered (Saari, 1989). DMSO significantly reduced copper deficiencyinduced heart hypertrophy, but did not prevent its occurrence. An argument has been made that the reduced heart hypertrophy may not result directly from DMSO, because the decreased food intake associated with DMSO administration may have contributed to the observed cardiac effect (Medeiros and Wildman, 1997). However, the lack of an unequivocal experimental approach makes this question difficult to answer.
In recent studies we have produced transgenic mice in which metallothionein (MT) is specifically overexpressed in the heart without compromising any other antioxidant systems (Kang et al, 1997). MT is a highly conserved, low-molecular-weight, thiol-rich protein. The mammalian MT has 61 amino acids, including 20 cysteine residues, but no aromatic amino acids, histidine or leucine. The basal level of MT in biological systems is very low, although it may vary with age and type of tissue (Hamer, 1986). However, this protein is highly inducible when the system is challenged by heavy metals, starvation, heat, inflammation, or other stress conditions (Kagi, 1991). Several in vitro studies have indicated that MT plays an important role in scavenging free radicals produced under various stress conditions (Sato and Bremner, 1993). Using the cardiac-specific, MT-overexpressing, transgenic mouse model, we have demonstrated that MT elevation makes the heart highly resistant to oxidative injury by adriamycin, an anticancer drug (Kang et al, 1997), and by ischemia and reperfusion (Kang et al, 1999). This unique experimental model thus provides a very valuable tool to study the role of oxy-radicals in cardiomyopathy induced by copper deficiency.

In the present study, the cardiac-specific, MToverexpressing, transgenic mouse model was used to determine the effect of MT on copper deficiencyinduced heart hypertrophy. Cellular and molecular events occurring during the progression of cardiac hypertrophy were investigated. In particular, the induction of apoptosis by copper deficiency and the role of atrial natriuretic peptide (ANP) in the apoptotic process were examined. Novel observations were obtained and the data demonstrate that elevation of MT in the heart inhibits oxidative injury, suppresses the progress of heart hypertrophy caused by copper deficiency, and most likely inhibits the transition from heart hypertrophy to failure by suppressing apoptosis, which is mediated at least in part by ANP production.

\section{Results}

\section{Systemic Effects of Dietary Copper Restriction}

To confirm the occurrence of organ copper depletion by dietary copper restriction, tissue copper concentrations, serum ceruloplasmin, and hepatic $\mathrm{Cu}, \mathrm{Zn}$ SOD activities were measured. Table 1 shows that heart and liver copper concentrations significantly

Table 1. Changes in Cu Concentrations in the Heart and Liver of MT-TG (MT') and Non-TG (MT') Mice

\begin{tabular}{|c|c|c|c|c|c|c|}
\hline & \multicolumn{3}{|c|}{ Heart ( $\mu \mathrm{g} / \mathrm{g}$ dry wt) } & \multicolumn{3}{|c|}{ Liver ( $\mu \mathrm{g} / \mathrm{g}$ dry wt) } \\
\hline & wk 3 & wk 4 & wk 5 & wk 3 & wk 4 & wk 5 \\
\hline CuA/MT ${ }^{-}$ & $21.8 \pm 2.1$ & $23.8 \pm 0.6$ & $25.1 \pm 2.7$ & $39.2 \pm 8.2$ & $38.7 \pm 3.1$ & $37.8 \pm 1.4$ \\
\hline $\mathrm{CuA} / \mathrm{MT}^{+}$ & $36.8 \pm 1.6^{*}$ & $35.5 \pm 1.9^{*}$ & $35.7 \pm 0.8^{*}$ & $38.9 \pm 9.9$ & $36.9 \pm 1.2$ & $39.6 \pm 5.4$ \\
\hline $\mathrm{CuD} / \mathrm{MT}^{-}$ & $15.7 \pm 0.8^{*}$ & $9.7 \pm 1.3^{*}$ & $7.1 \pm 3.3^{*}$ & $10.5 \pm 2.5^{\star}$ & $5.6 \pm 0.4^{*}$ & $5.6 \pm 1.3^{*}$ \\
\hline $\mathrm{CuD} / \mathrm{MT}^{+}$ & $15.8 \pm 4.7^{\star}$ & $8.4 \pm 0.7^{*}$ & $5.1 \pm 0.5^{\star}$ & $10.1 \pm 0.8^{*}$ & $5.6 \pm 0.6^{*}$ & $5.1 \pm 1.7^{\star}$ \\
\hline
\end{tabular}

Values are means \pm SD $(n=6)$, CuA, copper-adequate; CuD, copper deficient.

* Significantly different from the CuA non-TG $\left(\mathrm{CuA} / \mathrm{MT}^{-}\right)$mice $(p<0.05)$ and significantly different from week $3(p<0.05)$. 
decreased in both MT-overexpressing transgenic (MT-TG) and non-transgenic (non-TG) mice after 3 weeks (pre- and post-weaning) of consuming the copper-deficient (CuD) diet, and further decreased after 4 and 5 weeks of copper deficiency. Copper concentrations in the hearts of MT-TG control mice were significantly higher than in the non-TG controls. Dietary copper deficiency, however, caused the same depressive effect on copper concentrations in both MT-TG and non-TG mouse hearts. The results shown in Table 2 demonstrate that serum ceruloplasmin concentrations were significantly decreased in both MT-TG and non-TG mice receiving CuD diet for 5 weeks. Hepatic Cu, Zn-SOD, not Mn-SOD activities, were also depressed in these animals. These results clearly demonstrate that dietary copper restriction caused systemic copper deficiency in these mice, regardless of transgenic status.

\section{Development of Heart Hypertrophy by Dietary Cu Deficiency}

The effects of MT on the progression of heart hypertrophy induced by dietary copper deficiency were determined (Fig. 1). MT concentrations in MT-TG mouse hearts were approximately 20 -fold higher than in non-TG hearts $(120.5 \pm 6.8$ vs. $5.6 \pm 0.8 \mu \mathrm{g} / \mathrm{g}$ tissue, respectively). Heart hypertrophy was determined by the ratio of heart weight to body weight. After 3 weeks, this ratio was not significantly different in the mice fed CuD and those fed copper-adequate (CuA) diet in either transgenic or non-transgenic animals. This ratio was significantly $(p<0.05)$ higher in CuD mice than in CuA mice after they were fed their respective diets for 4 weeks in both transgenic and non-transgenic mice. The difference in heart size between the CuD and CuA non-transgenic mice was further exaggerated after they were fed for 5 and 6 weeks. In contrast, this progression in heart hypertrophy caused by copper deficiency was inhibited in MT-TG mice (Fig. 1).

\section{Copper Deficiency-Induced Myocardial Apoptosis}

To further probe the effect of MT elevation on the progression of heart hypertrophy induced by copper deficiency, we examined cellular and molecular as-

Table 2. Changes in Hepatic Cu, Zn-SOD, and Mn-SOD, and Serum Ceruloplasmin in Mice Fed Copper-Deficient (CuD) Diet for 5 Weeks in Comparison with Those Fed Copper Adequate (CuA) Diet

\begin{tabular}{|c|c|c|c|}
\hline & $\begin{array}{l}\text { Cu,Zn-SOD } \\
\text { (U/mg protein) }\end{array}$ & $\begin{array}{c}\text { Mn-SOD } \\
\text { (U/mg protein) }\end{array}$ & $\begin{array}{l}\text { Ceruloplasmin } \\
\qquad(\mu \mathrm{g} / \mathrm{ml})\end{array}$ \\
\hline $\mathrm{CuA} / \mathrm{MT}^{-}$ & $150.9 \pm 8.7$ & $4.9 \pm 1.6$ & $109.3 \pm 14.3$ \\
\hline $\mathrm{CuA} / \mathrm{MT}^{+}$ & $145.1 \pm 11.1$ & $4.9 \pm 1.8$ & $112.5 \pm 9.6$ \\
\hline $\mathrm{CuD} / \mathrm{MT}^{-}$ & $88.5 \pm 12.6^{*}$ & $4.4 \pm 1.1$ & $23.7 \pm 9.6^{*}$ \\
\hline $\mathrm{CuD} / \mathrm{MT}^{+}$ & $90.5 \pm 9.2^{*}$ & $4.0 \pm 1.4$ & $31.0 \pm 7.9^{*}$ \\
\hline
\end{tabular}

* Significantly different from controls (both CuA/MT ${ }^{-}$and $\mathrm{CuD} / \mathrm{MT}^{+}, p<$ 0.05).

Data are expressed as mean \pm SD $(n=6)$.

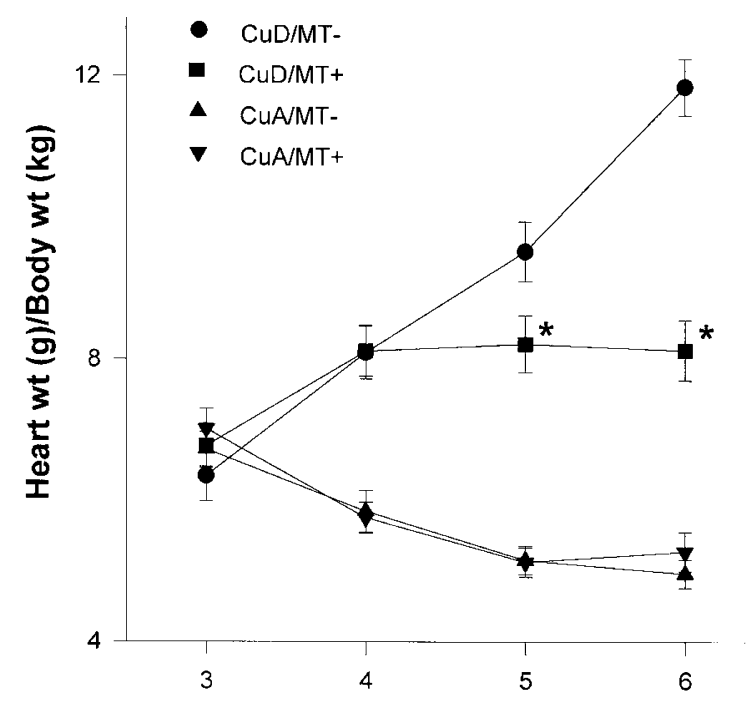

Week

Figure 1.

Effect of metallothionein (MT) on copper deficiency-induced heart hypertrophy in mice. Animals were fed a copper-deficient (CuD) diet or copper-adequate (CuA) diet for a total of 6 weeks (combined pre- and post-weaning). The hearts were harvested every week after they were fed for 3 weeks. Values are expressed as means \pm SD. Each value was obtained from 10 to 23 animals $(n=10$ to 23$)$. ${ }^{*}$ Indicates significant difference from other treatment groups $(p<0.05)$.

pects of heart hypertrophy. It has been recognized that the loss of cardiac myocytes is a fundamental part of the myocardial process that initiates and/or aggravates the transition from heart hypertrophy to heart failure (Lorell, 1997). An important aspect of the loss of myocytes is its mediation by apoptosis (Sabbah and Sharov, 1998), which has been demonstrated in the myocardium of heart failure patients (Olivetti et al, 1997). We examined apoptosis in the hearts of mice fed CuD diet for $24,36,42$, or 57 days. The results shown in Figure 2 demonstrate by a TUNEL assay that copper deficiency induced apoptosis in the mouse heart and MT significantly inhibited this apoptotic effect. To identify whether this apoptosis occurred in myocytes, an additional immunohistochemical staining for cardiac a-sarcomeric actin was performed. As shown in Figure $2 \mathrm{H}$, most of the TUNEL-positive cells were reactive to the monoclonal anti- $\alpha$-sarcomeric actin antibody, demonstrating that they were myocytes.

\section{Effect of MT on Copper Deficiency-Induced Lipid Peroxidation}

To determine whether the inhibitory effect of MT on the progression of heart hypertrophy induced by copper deficiency is related to MT inhibition of oxidative injury in the heart, malondialdehyde (MDA), a product of lipid peroxidation that is often used as an indictor of oxidative injury, was measured in the heart. As shown in Figure 3, free MDA concentration in the heart was markedly elevated in non-TG mice fed CuD diet for 5 


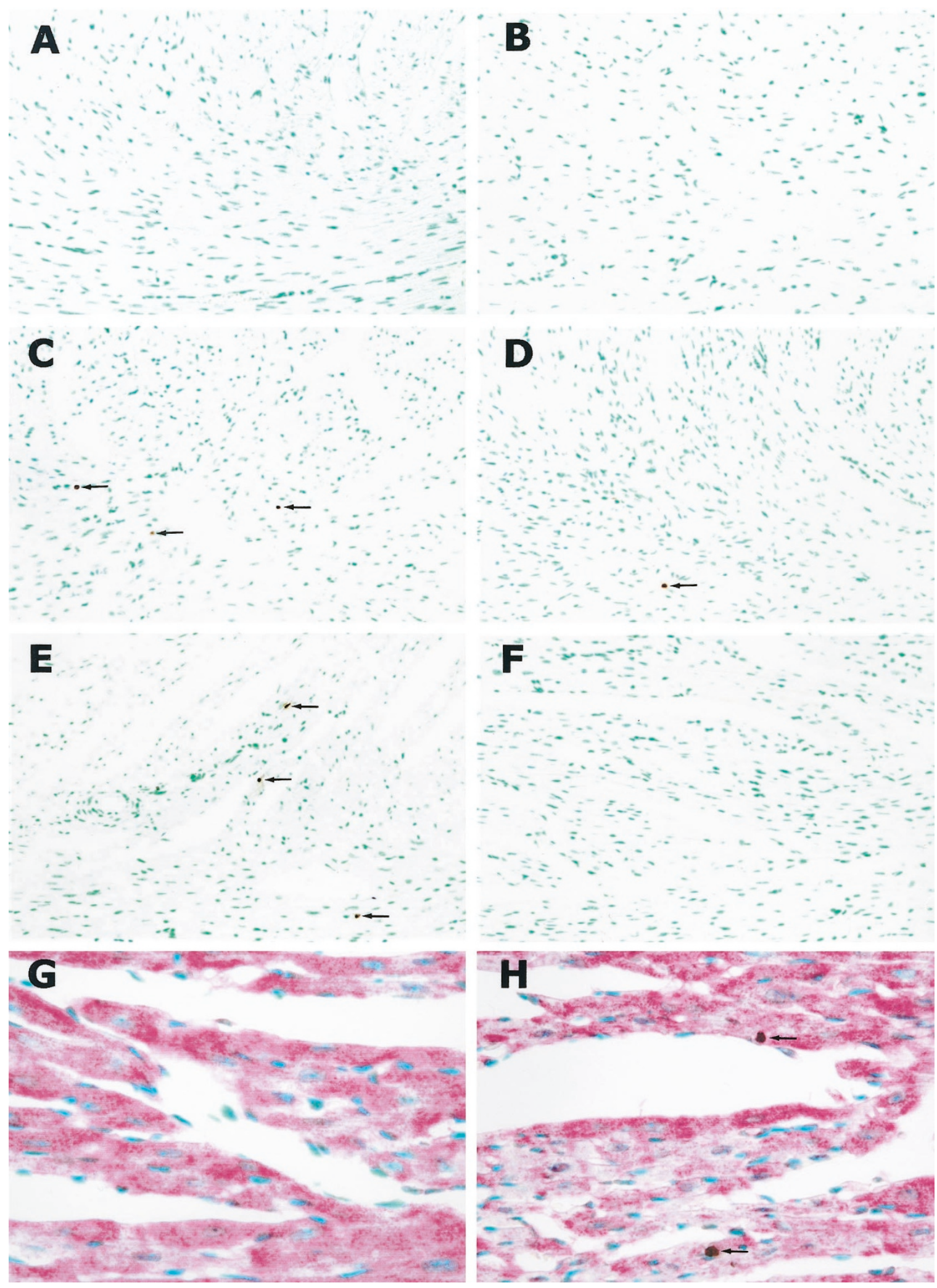

\section{Figure 2.}

Effect of MT on Cu-deficiency-induced apoptosis in the heart detected by a TUNEL assay (A to H). Myocardial tissues obtained from both non-transgenic (A) and transgenic (B) mice fed CuA diet were TUNEL negative. Apoptotic cells (arrows) in myocardial tissues obtained from both non-transgenic ( $\mathrm{C}$ and $\mathrm{E})$ and transgenic mice ( $\mathrm{D}$ and $\mathrm{F}$ ) fed $\mathrm{CuD}$ diet for $24(\mathrm{C}$ and $\mathrm{D}$ ) or 42 days ( $\mathrm{E}$ and $\mathrm{F}$ ) are shown. Immunohistochemical staining for cardiac $\alpha$-sarcomeric actin in non-transgenic myocardium $(\mathrm{G})$ and dual staining apoptotic cardiomyocytes in CuD non-transgenic myocardium as indicated by the arrow $(\mathrm{H})$. The graph in (I) shows the data were semi-quantified by counting the apoptotic nuclei against normal nuclei. The data were obtained from 3 animals for each treatment and 3 randomly selected fields (total of 1000 nuclei) were counted on each slide from each animal. The mean was obtained from the pooled averaged numbers from the 3 animals. * indicates significantly different from controls. A to $\mathrm{F}$, original magnification $\times 90$; $\mathrm{G}$ and $\mathrm{H}$, original magnification $\times 500$. 


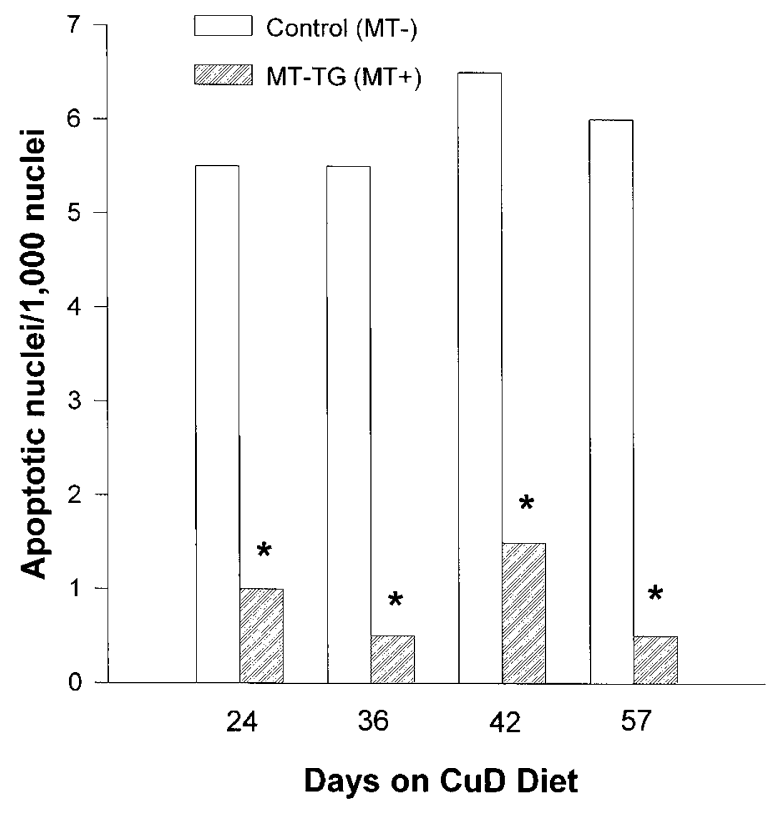

Figure 21.

weeks, and this elevation was significantly inhibited in MT-TG hearts.

\section{Effect of MT on Myocardial ANP Production Induced by Copper Deficiency}

One of the molecular features of the transition from heart hypertrophy to heart failure is the production of atrial natriuretic peptide (ANP) in the left ventricle. The ANP gene is expressed in both atrium and ventricle during embryonic development, but its expression is

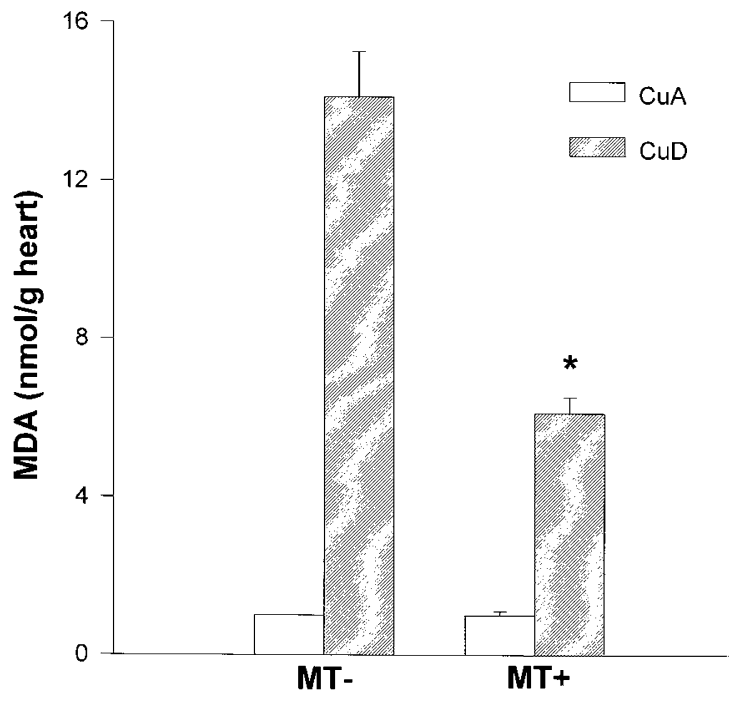

Figure 3.

Effect of MT on free malondialdehyde (MDA) concentrations in CuD mouse hearts. Animals fed CuD or CuA diet for 5 weeks were killed and the hearts removed as described in Materials and Methods. The freshly-removed hearts were immediately processed for the isolation of free MDA and measurement by an HPLC system. Each value was obtained from 10 to 12 animals ( $n=10$ to 12 ) and the data are expressed as mean $\pm \mathrm{SD} .{ }^{*}$ indicates significantly different from CuA. down-regulated in the ventricle shortly after birth, leaving the atrium as the primary site of ANP synthesis within the mature myocardium. During the progression of heart hypertrophy, in particular, the transition from hypertrophy to failure, re-expression of ANP in myocytes of the left ventricle occurs (Yasue et al, 1994). Detecting the abundance of mRNA for ANP in the left ventricle is thus an important index of the transition. The long-term expression of ANP is associated with numerous detrimental biological effects that eventually lead to cardiac failure by way of myocyte dysfunction, apoptosis, and cell loss. The expression of ANP in the left ventricle of mice fed CuD diet for 5 weeks was therefore determined. As shown in Figure 4 and Table 3, copper deficiency caused a significant elevation of the abundance of mRNA for ANP in the mouse heart. This elevation was markedly depressed in MT-TG mouse hearts.

\section{Effect of MT on ANP-Induced Apoptosis in Cultured Cardiomyocytes}

Although the correlation between overexpression of ANP and apoptosis in the heart by copper deficiency was positive, it is important to know whether ANP is responsible for the apoptosis in the heart. In this context, we applied a newly-established primary neonatal cardiomyocyte culture system (Wang and Kang, 1999; Wang et al, 1999). Cardiomyocytes, after being cultured for 4 days on special cell culture slides, were challenged with different concentrations of ANP for 4 hours. As shown in Figure 5, ANP markedly induced apoptosis in both dose-dependent and time-related fashions, as revealed by the TUNEL assay. Importantly, MT-TG cardiomyocytes were significantly resistant to the ANP-induced apoptosis.

To confirm the results obtained from the TUNEL assay, a more sensitive early detection method for apoptosis, Annexin V-FITC staining and confocal microscopy, was used. The results presented in Figure 6 show the time course studies of the cells exposed to $0.1 \mu \mathrm{M}$ ANP. Annexin V-FITC-positive cells were detected as early as 1 hour after ANP exposure in non-TG cultures. No positive cells were detected in MT-TG myocyte cultures at any time point of expo-

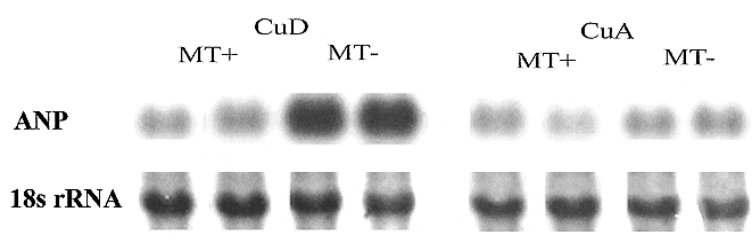

Figure 4.

Effect of MT on elevation of ANP mRNA by copper deficiency in mouse hearts. Mice were fed either CuD or CuA diet for 5 weeks. The left ventricle was then removed from the killed mice. The abundances of mRNAs for ANP was measured 5 times with 2 to 3 animals at each time and consistent results were obtained. The representative autoradiography presents the result obtained from 1 experiment with 2 animals for each group. The data obtained from all experiments by an autoradiographic image analysis system were calculated and the arbitrarily quantitative data (label intensity analyzed by computer) were compared between $\mathrm{CuD}$ and CuA groups and expressed as means \pm SD shown in Table 3. 
Table 3. Effect of MT on Expression of ANP mRNA by Copper Deficiency ${ }^{a}$

\begin{tabular}{lccc}
\hline & CuD & CuA & CuD/CuA \\
\hline Non-TG (MT-) & $96.4 \pm 0.6$ & $27.1 \pm 2.3$ & 3.56 \\
MT-TG (MT+) & $26.2 \pm 1.4^{*}$ & $18.7 \pm 3.5$ & $1.40^{*}$ \\
\hline
\end{tabular}

${ }^{a}$ Follow Figure 4.

* Significantly different from the non-TG mice $(p<0.05)$.

Values are means \pm SD $(n=10-15)$

sure. A dose-dependent effect of ANP was also determined. After 4 hours of treatment, cells stained positively for Annexin V-FITC were not found in non-TG myocyte cultures exposed to $0.01 \mu \mathrm{M}$ ANP, were highly detectable in non-TG cultures exposed to $0.1 \mu \mathrm{M}$, and were moderately detectable in non-TG cultures exposed to $1.0 \mu \mathrm{m}$. Positive cells were not detected at any dose level in MT-TG cell cultures.

\section{Discussion}

Heart hypertrophy induced by dietary $\mathrm{Cu}$ restriction has been shown to be comparable to that induced by pressure overload in many aspects, including morphological, biochemical, and physiological alterations (Medeiros et al, 1993; Prohaska, 1990). Extensive studies have been undertaken to understand the progression of cardiac hypertrophy induced by pressure overload. The transition from heart hypertrophy to failure is an important area of this research. This transition has not been previously addressed for the case of cardiac hypertrophy induced by copper deficiency.

In the present study, the time course of heart hypertrophy induced by dietary copper deficiency was examined. Mice fed CuD diet for 4 weeks showed significant heart hypertrophy that progressed as the feeding continued. This progression was associated with a significant myocardial cellular complication, apoptosis. This is a critical event in the transition from heart hypertrophy to failure (Lorell, 1997; Sabbah and Sharov, 1998). The identification of elevated myocardial apoptosis with the progression of heart hypertrophy thus indicates that this transition occurs in the copper-deficient heart.

The TUNEL assay used in the present study identifies any apoptotic cells in the myocardium. To determine whether apoptosis occurred in myocytes, TUNEL-stained myocardial tissue was also stained with anti-cardiac $\alpha$-sarcomeric actin antibody. $\alpha$-Sarcomeric actin is a specific protein present in the cardiomyocytes. This staining thus identifies cells of myocyte origin. Significant numbers of cells were dually stained by the TUNEL and the anti- $\alpha$ sarcomeric actin antibody, demonstrating that copper deficiency-induced apoptosis did occur in cardiomyocytes.

It is important to stress that the apoptosis observed in the copper-deficient heart would lead to very significant consequences in terms of the loss of myocytes. In a carefully designed time course study (Ka- jstura et al, 1996), it has been estimated that cardiomyocyte apoptosis may be completed in less than 20 hours in rats. Because the heart is a terminally differentiated organ, myocytes undergoing apoptosis will be lost and are not replaced. Thus the loss of cells can simply be accounted for by the rate of apoptosis plus any other losses. If apoptosis occurs at a constant rate of approximately $0.5 \%$ myocytes a day, as estimated from the present study, the potential contribution of apoptosis to the overall loss of myocytes in copper deficiency is accountable.

That MT inhibited the progression, but not the initiation, of heart hypertrophy induced by copper deficiency has a significant impact on studies of the resultant cardiomyopathy. In particular, the observation that the inhibition of myocardial apoptosis in the MT-TG mice correlated with the suppression of lipid peroxidation in the heart by MT provides progress toward understanding the role of oxy-radicals and antioxidants in the cardiomyopathy. It demonstrates for the first time that the active site of antioxidants is not at the initiation, but rather during the progression of the hypertrophy. This provides a more specific area for research aimed at understanding the relationship between oxy-radicals and the cardiomyopathy induced by copper deficiency.

Several studies using rat and guinea pig models have revealed that antioxidant capacity was depressed and oxidative injury increased during the transition from heart hypertrophy to failure (Dhalla and Singal, 1994; Gupta and Singal, 1989; Hill and Singal, 1996, 1997). In these studies, the left coronary artery was ligated at 1 to $2 \mathrm{~mm}$ from its origin. This procedure produced approximately $35 \%$ mortality within 24 hours after ligation. The surviving animals developed heart hypertrophy after acute myocardial infarction. The heart hypertrophy was followed by failure as determined by hemodynamic parameters (Dhalla and Singal, 1994; Hill and Singal, 1996, 1997). Interestingly, the activities of antioxidant systems, including SOD, catalase and GSH peroxidase, were all marginally or significantly increased in the non-failing hypertrophic hearts, but significantly depressed in the failing hearts (Dhalla and Singal, 1994; Gupta and Singal, 1989; Hill and Singal, 1996, 1997). An important consequence of the depression of antioxidants is the occurrence of lipid peroxidation. These studies lead to the hypothesis that a relative deficit in antioxidant reserves contributes to cardiac failure (Singal and Kirshenbaum, 1990; Singh et al, 1995).

To test this hypothesis is by no means an easy task. Supplementation of animals with antioxidants could be an approach to this issue. However, under in vivo conditions, three major problems complicate data interpretation: (1) it is impossible to maintain constant plasma antioxidant concentrations and to accurately predict the target tissue (heart) concentrations; (2) metabolic activation and inactivation by multiple metabolic organs such as liver and kidney would greatly affect the efficacy of the antioxidants; and (3) high molecular weight antioxidants such as superoxide dismutase, catalase, or GSH peroxidase are unlikely 

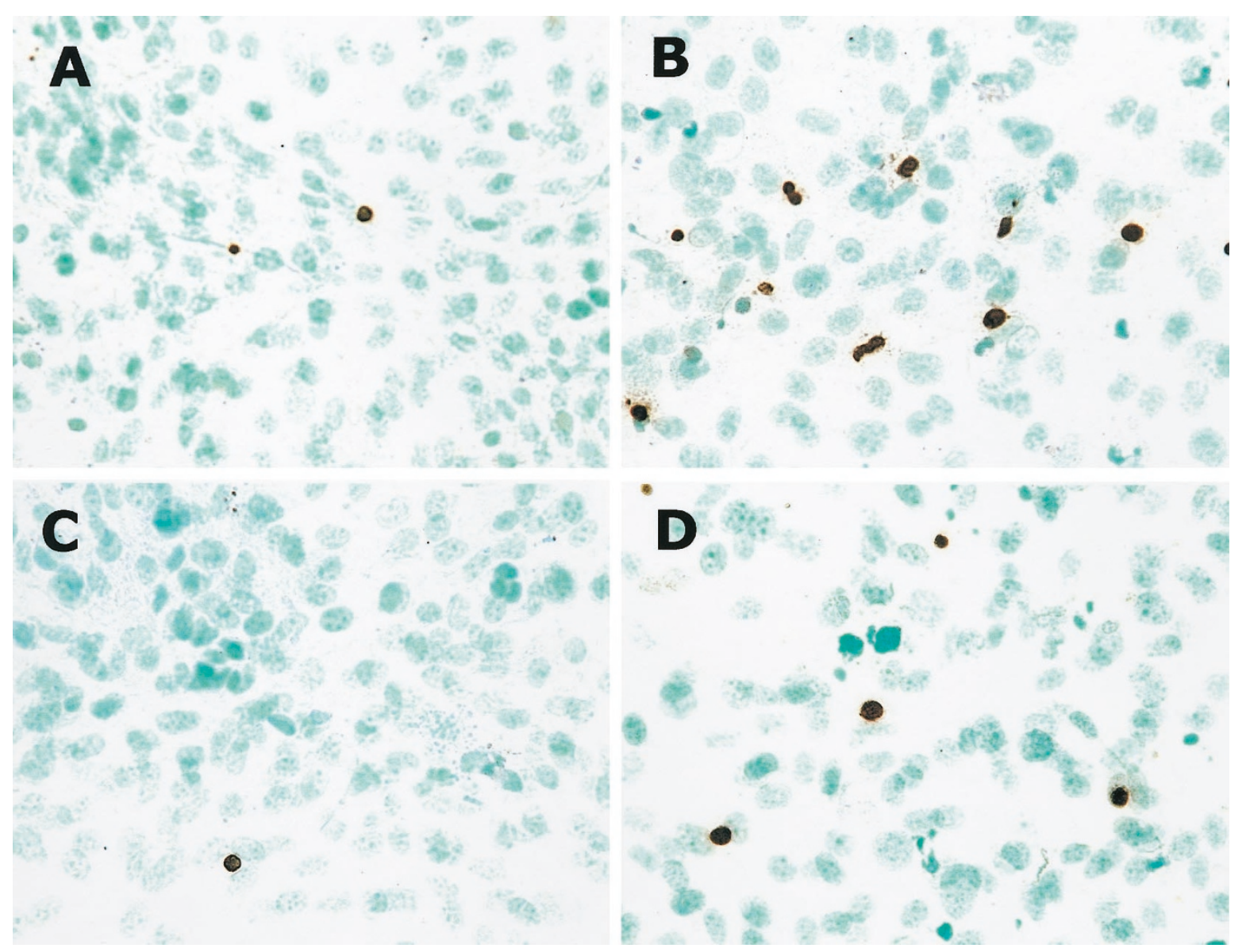

\section{Figure 5.}

Effect of MT on atrial natriuretic peptide- (ANP)-induced apoptosis in cultured primary mouse cardiac myocytes. Cultures of non-transgenic (A and B) and transgenic $(C$ and $D)$ cardiomyocytes were exposed to $0.1 \mu \mathrm{m}$ ANP for 4 hours (B and $D)$ and the apoptotic cells were detected by a TUNEL assay (A to D). The effect of MT on ANP concentration-dependent apoptotic effect was detected by the same TUNEL assay and semi-quantified by counting the apoptotic nuclei against normal nuclei (E). Data were obtained from 3 cultures for each treatment and are expressed as mean \pm SD. ${ }^{*}$ indicates significantly different from non-TG cultures. Original magnification $\times 370$.

to be transported into intracellular compartments. Furthermore, the required chronic and long-term studies of heart failure make these complications even worse. The transgenic mouse model that specifically overexpresses MT in the heart thus provides a unique tool to overcome these problems and the results obtained from this study strongly argue for the role of oxidative stress in the transition from heart hypertrophy to failure.

MT functions in Cu homeostasis, although this action of MT is poorly understood. The results presented in Table 1 show that dietary Cu deficiency caused the same depression in $\mathrm{Cu}$ concentrations in the heart between MT-TG and non-TG mice. Importantly, the time dependent kinetic changes in $\mathrm{Cu}$ concentrations in the heart (from week 3 to week 5) were also the same between MT-TG and non-TG. But the result presented in Figure 1 shows that the divergence of heart hypertrophy phenotype started to appear at week 5 . These results indicate that the difference in the changes in heart hypertrophy between MT-TG and non-TG mice cannot be explained by $\mathrm{Cu}$ concentrations in the heart. It is possible that a significant fraction of $\mathrm{Cu}$ is associated with $\mathrm{MT}$ and that the amount of this MT-associated $\mathrm{Cu}$ may be different between MT-TG and wild-type mice. However, this difference would not contribute to the different phenotype in the progression of heart hypertrophy. If there were any effect resulting from the difference in MTassociated $\mathrm{Cu}$, it would be an adverse one in the MT-TG mice because Cu association with MT would make it less available for other crucial cellular function. On the other hand, the results shown in Figure 1 correlate well with that in Figure 3 , showing that the MDA concentrations in the MT-TG mouse heart were significantly less than in the non-TG mouse heart, which is in agreement with the antioxidant function of MT (Kang, 1999).

Another important set of observations obtained from this study are the elevated production of ventricular ANP by copper deficiency and the evidence that it acts as a trigger for myocardial apoptosis. If apoptosis is a critical cellular event in the transition from heart hypertrophy to failure by dietary copper restriction, identification of the "trigger" is important in understanding the molecular mechanisms of this cardiomyopathy. ANP has long been used as a clinical indicator of heart failure, which suggests that it may be a trigger 


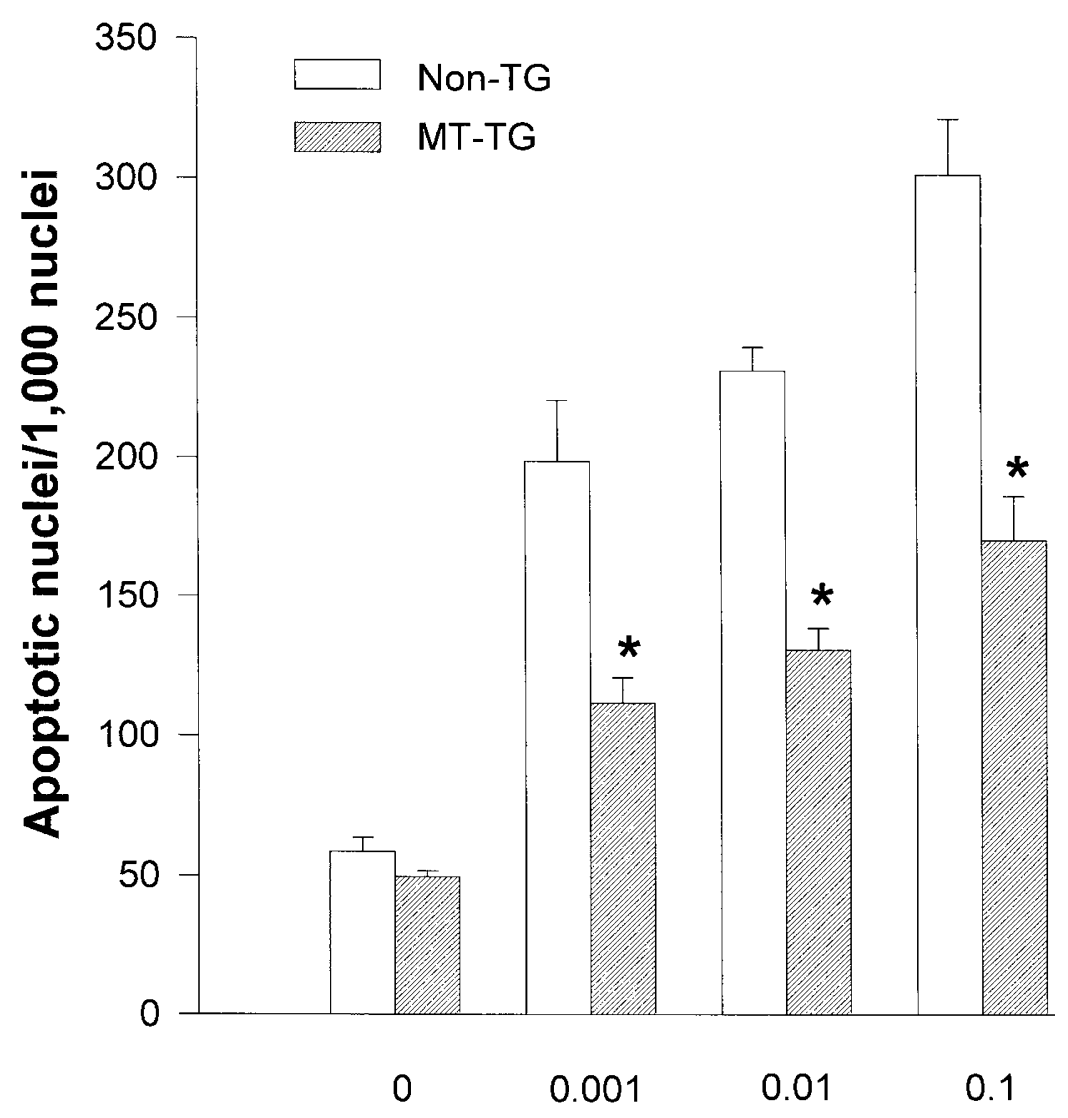

Figure 5E.

for myocardial apoptosis. Our finding of a correlation between ANP gene expression and apoptosis provided evidence that the two effects were related. This was revealed first, by the elevation of both myocardial apoptosis and ANP mRNA by copper deficiency, and second, by the suppression by MT of both ANP mRNA elevation and apoptosis. The cause-and-effect relationship between ANP production and myocyte apoptosis was then demonstrated in the studies using cardiomyocyte cultures. Time- and concentrationdependent apoptotic effects of ANP, measured by the TUNEL assay and Annexin V-FITC confocal microscopy, provided direct evidence for the induction of myocyte apoptosis by ANP.

MT inhibited both production of ANP in the left ventricle and its apoptotic effect. Whether oxidative stress up-regulates ANP production is unknown. However, the inhibitory effect of MT on lipid peroxidation suggests that antioxidant action may be an important mechanism by which MT inhibits ANP production. Recent studies have shown that ANP-induced apoptosis in neonatal rat cardiomyocytes is mediated by cGMP (Wu et al, 1997), which has been shown to increase nitric oxide (NO) concentrations (McAndrew et al, 1997). MT has been shown to function in detoxification of NO (Schwart et al, 1995), which may be a mechanism of the anti-apoptotic action of MT observed in the present study.

Based on the results of the present study and our previous observations, we speculate that MT may be useful in the prevention of heart failure. In particular, MT is highly inducible under a wide diversity of stress conditions. The regulation of MT expression has been well studied and several agents have been identified to selectively elevate MT levels in the heart, such as bismuth subnitrate (Naganuma, 1988), isoproterenol (Namikawa et al, 1993), and tumor necrosis factoralpha (Sato et al, 1992). Therefore, the basis for developing pharmaceutical agents to increase MT concentration in the heart already exists. Exploring the potential for MT to prevent heart failure would likely result in novel approaches to this clinical problem and could positively influence clinical outcomes.

Study of the transition from heart hypertrophy to failure is a major undertaking in cardiac research. Most animal models are produced by coronary artery ligation (Dhalla and Singal, 1994; Gupta and Singal, 1989; Hill and Singal, 1996, 1997). This procedure results in significant mortality $(>50 \%)$ in mice (unpublished observation by authors). The surviving mice live no more than 3 weeks, which does not allow heart failure to develop. An unexpected bonus of studying 


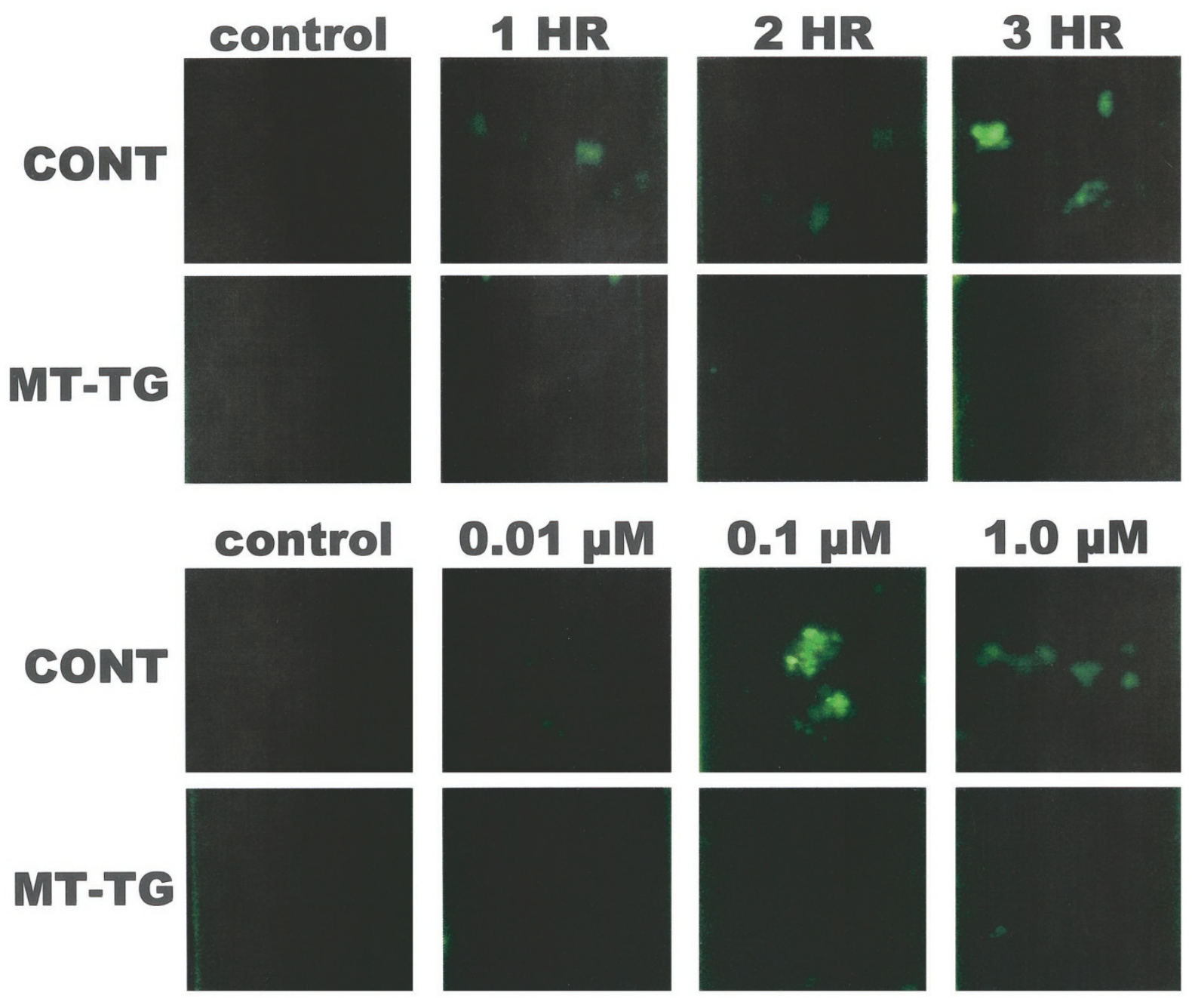

\section{Figure 6.}

Detection of apoptosis in cardiomyocyte cultures by Annexin-V-FITC staining. Cells were treated with $0.1 \mu \mathrm{M}$ ANP for varying time periods as indicated (top) and with different concentrations for 4 hours (bottom panel). Procedures for Annexin-V-FITC staining and confocal microscopic examination of positive cells were described in Material and Methods.

the cardiomyopathy by copper deficiency is that it provides an alternative approach to studying the transition from heart hypertrophy to failure. A great deal of similarity in heart hypertrophy induced by copper deficiency and pressure overload has been observed. Both cause concentric ventricular hypertrophy (Medeiros et al, 1993). The molecular aspects of myocardial remodeling between the two hypertrophied hearts are also similar (Kang et al, 2000). Thus, copperdeficient mice may well serve as an alternative experimental model for studying the transition from heart hypertrophy to failure. The unique aspects of copperdeficient mice are: (1) the mortality before heart failure is very low (less than 5\%), (2) the survival time is sufficient to allow detailed studies of transition from heart hypertrophy to failure, and (3) the unified feeding treatment significantly reduces the artifact due to coronary artery ligation surgery. Therefore, the copper-deficient animal model provides an alternative experimental approach to understanding the transition from heart hypertrophy to failure.

\section{Materials and Methods}

\section{Animals and Treatment}

FVB mice were bred and maintained at the University of Louisville animal facilities. The cardiac-specific, MT-overexpressing, transgenic (MT-TG) mice were produced from the FVB stain as described previously (Kang et al, 1997). The MT-TG mice were then bred with the same strain (FVB) of non-TG mice. They were housed in plastic cages at $22^{\circ} \mathrm{C}$ on a 12 hours light/dark cycle. Dams of the pups (both heterozygous MT-TG mice and their non-TG littermates) were fed copper-deficient (CuD) or copper-adequate (CuA) diet starting on the fourth day post delivery. The pups were weaned on the 21st day after birth and the weanling mice were continued on the same diet as their dams until they were killed at week 3,4 , or 5 weeks after CuD feeding (combined pre- and post-weanling feeding). The animals had free access to doubly distilled water. Body weight gain of each mouse was moni- 
tored once a week during the experiment. The CuA and CuD diets (AIN-93 diet) were prepared according to Reeves et al (1993) and the primary ingredients were cornstarch (53\%), casein (20\%), sucrose (10\%), and soybean oil (7\%). Vitamins and minerals were provided in the diet. The CuA diet included an addition of $6 \mathrm{mg}$ of $\mathrm{Cu} / \mathrm{kg}$ diet, and the corresponding weight of cornstarch was added to the CuD diet. Analyses of the diets for $\mathrm{Cu}$ concentration yielded $6.089 \mathrm{mg} \mathrm{Cu} / \mathrm{kg}$ diet for CuA and $0.348 \mathrm{mg} \mathrm{Cu} / \mathrm{kg}$ diet for CuD diet. All procedures were approved by the AAALAC certified University of Louisville Institutional Animal Care and Use Committee.

\section{Tissue Harvest}

At the end of the feeding experiment and after an overnight fast, each animal was anesthetized with an intraperitoneal injection of sodium pentobarbital (65 $\mathrm{mg} / \mathrm{kg}$ body weight; Vet Labs, Lenexa, Kansas). Blood was withdrawn from the abdominal vena cava and serum was separated with a Serum Separator (Becton Dickenson, Rutherford, New Jersey) within 30 minutes. An incision was made in the inferior vena cava and the heart was perfused with cold $0.9 \% \mathrm{NaCl}$. The heart was then removed, opened, washed, dried with paper tissue, and weighed. Part of the left ventricle was used for mRNA analysis and the remainder of the heart was used for mineral and enzymatic determinations. The liver was also perfused with cold $0.9 \% \mathrm{NaCl}$ through the portal vein and portions of liver were excised. All the tissue samples were either used immediately or placed in liquid nitrogen, then stored at $-80^{\circ} \mathrm{C}$ for no longer than 36 hours before analysis.

\section{Cu Concentrations}

$\mathrm{Cu}$ concentrations in the heart and the liver were measured using inductively coupled argon plasma emission spectroscopy (model 35608, Thermo ARL-VG Elemental, Franklin, Massachusetts) after lyophilization and digestion of the tissues with nitric acid and hydrogen peroxide (Nielsen et al, 1982). Dietary $\mathrm{Cu}$ concentrations were analyzed by using a dryashing procedure, which was followed by dissolution of the residue in aqua regia and measurement by atomic absorption spectrophotometry (model 503; Perkin Elmer, Norwalk, Connecticut). Trace element contents of National Institute of Standards and Technology (NIST; Gaithersburg, Maryland) reference samples were within the specified ranges established by NIST, thus validating our assay procedure.

\section{Serum Ceruloplasmin}

Serum ceruloplasmin concentrations were determined by its p-phenylenediamine (PPD) oxidase activity (Sunderman and Nomoto, 1970). The oxidation of PPD at $\mathrm{pH} 5.4$ yields a product that is readily detectable colorimetrically at $530 \mathrm{~nm}$. The rate of product formation is proportional to the concentration of ceruloplas$\min$.

\section{Cu, Zn-Superoxide Dismutase (Cu, Zn-SOD)}

Total SOD activity was determined by a NBT assay according to Spitz and Oberley (1989). Briefly, tissue samples were homogenized in 19 volumes of $0.5 \mathrm{M}$ potassium phosphate buffer, $\mathrm{pH}$ 7.8. After centrifugation at $700 \mathrm{~g}$ at $4^{\circ} \mathrm{C}$ for 10 minutes, the supernatant was collected and diluted 20 -fold, and $50 \mu \mathrm{l}$ of the diluted supernatant were applied to the assay. In each 1-ml assay cuvette, the following were added with the indicated final concentrations: potassium phosphate buffer (50 mM), pH 7.8; DETAPAC (1.0 mm); catalase (1.0 unit); NBT $\left(5.6 \times 10^{-5} \mathrm{M}\right)$; xanthine $(0.1 \mathrm{~mm})$. To initiate the reaction, xanthine oxidase (50 $\mu$ l of 0.08 $\mathrm{U} / \mathrm{ml}$ ) was added. Absorbance at $560 \mathrm{~nm}$ was monitored for 6 minutes with a spectrophotometer (Beckman DU-650, Fullerton, California). This procedure was also performed in the presence of $\mathrm{NaCN}(5 \mathrm{~mm})$ to assay for Mn-SOD activity and the $\mathrm{Cu}, \mathrm{Zn}$-SOD was calculated by subtracting the Mn-SOD activity from the total SOD activity.

\section{Metallothionein}

Total tissue MT concentrations were determined by the cadmium-hemoglobin affinity assay (Eaton and Cherian, 1991). Briefly, tissues were homogenized in 4 volumes of $10 \mathrm{~mm}$ Tris- $\mathrm{HCl}$ buffer, $\mathrm{pH} 7.4$ at $4^{\circ} \mathrm{C}$. After centrifugation of the homogenate at $10,000 \mathrm{~g}$ for 15 minutes, $200 \mu$ l supernatant was transferred to microtubes for MT analysis as described previously (Kang et al, 1997).

\section{Measurement of Lipid Peroxidation}

Malondialdehyde (MDA) is a major product of lipid peroxidation induced by oxidative stress. A commonly used method to estimate MDA concentrations in tissue is the thiobarbituric acid (TBA) test. However, this colorimetric method is not specific for MDA, because other known and unknown compounds produce positive reactions and the presence of transition metals significantly affects the results. It is questionable whether the TBA value can be used as a reliable index for lipid peroxidation. In biological systems, the cytotoxic aldehydes, including MDA, are extremely active. They can diffuse within, or even escape from, the site of the original free radical initiated event, and therefore act as "second cytotoxic messengers" (Esterbauer and Zollner, 1989; Loidl-Stahlhofen and Spiteller, 1994). Although it is challenging, measuring free MDA in the heart before it is bound to biological macromolecules is a meaningful means to explore the mechanism involved in copper deficiency-induced cardiac lipid peroxidation. A modified HPLC method (Ceconi et al, 1992; Wu and Kang, 1998) was therefore adopted to measure free MDA concentrations in the hearts. The HPLC separations were performed on a Val-U-Park $\mathrm{C}_{18}$ column $(0.46 \times 25)$ (Supelco, Bellefonte, Pennsylvania) with a mobile phase of $10 \mathrm{~mm}$ $\mathrm{Na}_{2} \mathrm{HPO}_{4}, 2.5 \mathrm{~mm}$ myristlyltrimethyl-ammonium bromide, $25 \%$ acetonitrile ( $\mathrm{pH} 6.7$ ), at a flow-rate of 0.8 $\mathrm{ml} /$ minute and at ambient temperature. The injected 
volume was $20 \mu \mathrm{l}$. The HPLC instrument was a Waters series delivery system (Milford, Massachusetts), and the detector wavelength was $267 \mathrm{~nm}$. A $0.5 \mu \mathrm{M}$ MDA, standard solution was made by diluting $10 \mathrm{~mm}$ MDA which was prepared from malonaldehydebisdiethylacetal by acid acidic hydrolyzing and then dissolving in $1.15 \% \mathrm{KCl}$ - acetonitrile. The concentration was checked by measuring the UV absorbance in 1-cm cuvettes at $245 \mathrm{~nm}$ (Esterbauer et al, 1984).

\section{Neonatal Mouse Primary Cardiomyocyte Culturing}

A new procedure for culturing ventricular cardiomyocytes from neonatal mice was described previously (Wang et al, 1999). Briefly, 1- to 2-day old neonatal transgenic or nontransgenic mice were killed by cervical dislocation. Hearts were removed aseptically (retaining the ventricles only) and kept in cold Hanks buffered saline solution (HBSS) without $\mathrm{Ca}^{2+}$ and $\mathrm{Mg}^{2+}$. The ventricles were washed with the same HBSS and minced into small fragments. Cardiac cells were dissociated with a trypsin solution and obtained by centrifugation. To exclude non-muscle cells, the isolated cells were first plated in tissue culture dishes at $37^{\circ} \mathrm{C}$ for 2 hours under a water-saturated atmosphere of $5 \% \mathrm{CO}_{2}$ with $95 \%$ air. The suspended cells (myocytes) were then collected and plated at a density of $1.0 \times 10^{5}$ cells $/ \mathrm{cm}^{2}$ in $100-\mathrm{mm}$ culture dishes or on glass cover-slips, and incubated under the same conditions as above. Myocyte purity was monitored by staining with antibody to cardiac-sarcomeric actin according to the manufacturer's instructions (Sigma, St. Louis, Missouri) and was found to average $94 \pm$ $5 \%$ when examined at 48 hours after culturing.

\section{Determination of Copper Deficiency-Induced Apoptosis In Vivo}

The hearts removed from copper-deficient and control mice were fixed in $10 \%$ formalin in $0.01 \mathrm{M}$ phosphate buffered saline, $\mathrm{pH} 7.3$, for 24 hours and embedded in paraplast. Tissue blocks were sectioned at a thickness of $5 \mu \mathrm{m}$ and mounted on silanized slides.

\section{TUNEL Assay}

The slides obtained were processed for a TUNEL assay to detect fragmented nuclei in the myocardium. An ApopTag in situ detection kit (Intergen, Purchase, New York) was used according to the manufacturer's instruction. Briefly, the slides were pretreated with $\mathrm{H}_{2} \mathrm{O}_{2}$ and incubated with the reaction mixture containing TdT and digoxigenin-conjugated dUTP for 1 hour at $37^{\circ} \mathrm{C}$. Labeled DNA was visualized with peroxidase-conjugated anti-digoxigenin antibody with $\mathrm{DAB}$ as the chromagen. Rat mammary gland tissue provided in the kit was used as positive control. For negative control, TdT was routinely omitted from the reaction mixture.

\section{Dual Staining of Myoglobin}

After the TUNEL staining, some of the tissue sections were processed for $\alpha$-sarcomeric actin localization with an immunoperoxidase method. Sections were incubated with monclonal $\alpha$-sarcomeric actin (Clone $5 \mathrm{C} 5$, Isotype mouse IgM; Sigma) overnight at $4^{\circ} \mathrm{C}$, then with biotinylated rabbit anti-mouse IgM (Zymed Laboratory, San Francisco, California), followed by streptavidin-alkaline phosphatase (Zymed) incubation. A red chromagen of new fuchsin was used to visualize the binding sites.

\section{Determination of Apoptosis in Cultured Cardiomyocytes}

Cardiomyocytes cultured on glass cover-slips were washed with PBS and fixed in 1\% paraformaldehyde for 10 minutes and post-fixed in pre-cooled ethanol:acetic acid (2:1) for another 5 minutes at $-20^{\circ} \mathrm{C}$. After washing with PBS, the cells were incubated with the TUNEL reactive mixture for 1 hour at $37^{\circ} \mathrm{C}$ in a humidified chamber. As a positive control, cells were treated with DNase I ( $1 \mu \mathrm{g} / \mathrm{ml}$, Sigma) for 10 minutes to introduce nicks in the genomic DNA. The percentage of myocytes with DNA nick end-labeling was determined by counting cells exhibiting brown nuclei (TUNEL positive) among 10,000 nuclei in triplicate plates. ANP was added by replacing the culturing media with fresh ANP containing media with a designated concentration. After ANP treatment, media were removed from the culturing slides and the slides were stained by the TUNEL assay. The proportion of apoptotic cells relative to the total number of cells was estimated by counting 1000 nuclei from at least 5 fields of each slide and using 3 slides for each concentration of ANP.

To confirm the TUNEL determination of apoptotic cardiomyocytes in cultures, Annexin V-FITC binding was determined by confocal microcopy. Cells undergoing apoptosis lose plasma membrane asymmetry and phospholipid phosphatidylserine (PS) is translocated from the inner leaflet of the plasma membrane to the outer leaflet, thereby exposing PS to the exter-

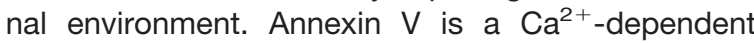
phospholipid-binding protein that has a high affinity for PS, and is useful for identifying apoptotic cells with exposed PS. Cells that are stained positive for Annexin V-FITC are in the early stages of apoptosis. Cells that were cultured on glass cover-slips were washed with proprietary binding buffer and stained with FITCconjugated Annexin V (Immunotech, Fullerton, California) for 10 minutes. The samples were then optically sectioned with a confocal microscopy system equipped with a Nikon MT-2 microscope.

\section{Northern Blot Analysis}

Total RNA was extracted from left ventricles of mice using a RNAzol B method. RNA was quantified spectrophotometrically and confirmed by ethidium bromide staining of $18 \mathrm{~S}$ and $28 \mathrm{~S}$ ribosomal RNA. RNA was denatured in formaldehyde, fractionated by electrophoresis on $1.0 \%$ agarose gels, and transferred to nylon membranes. The oligonucleotide used as a transcript specific probe for ANP was as follows: 5'-AATGTG-ACCAAGCTGCGTGACACACCACAAGG- 
GCTTAGGATCTTTTGCGATCTGCTCAAG. The probe was labeled with $\left[{ }^{32} \mathrm{P}\right] \mathrm{dATP}$ using $\mathrm{T}_{4}$ kinase (NEN, Boston, Massachusetts) and purified and precipitated by salt and ethyl alcohol. Hybridization and wash procedures were conducted using previously published methods (Church and Gilbert, 1984). Autoradiographic images were scanned and analyzed using an MCID system from Imaging Research (St. Catharines, Ontario, Canada). The data were quantified and compared between groups by a computerized densitometric analysis of the label intensity.

\section{Statistical Analysis}

Data were analyzed initially by two-way ANOVA, but the data presented in Table 1 were analyzed by three-way ANOVA. Scheffe's F-test was employed for further determination of the significance of differences. Differences between MT overexpressing transgenic mice and non-transgenic controls were considered significant at $p<0.05$. The data are presented as mean $\pm S D$ values from the indicated number of animals or cultures for each treatment.

\section{Acknowledgements}

The authors thank Donald Mosley, Kristie Lock, Angela Mitchell, Gwen Dahlen and Peter Leary for technical assistance. YJK is a University Scholar of the University of Louisville.

\section{References}

Akers TK, and Saari JT (1993). Hyperbaric hyperoxia exaggerates respiratory membrane defects in the copperdeficient rat lung. Biol Trace Elem Res 38:149-163.

Balevska PS, Russanov EM, and Kassabova TA (1981). Studies on lipid peroxidation in rat liver by copper deficiency. Eu J Biochem 13:489-493.

Bode AM, Miller LA, Faber J, and Saari JT (1992). Mitochondrial respiration in heart, liver, and kidney of copper-deficient rats. J Nutr Biochem 3:668-672.

Borg TK, Klevay LM, Gay RE, Siegel R, and Bergin ME (1985). Alteration of the connective tissue network of striated muscle in copper-deficient rats. J Mol Cell Cardiol 17:1173-1183.

Ceconi C, Cargnoni A, Pasini E, Cordorelli E, Curello S, and Ferrari R (1992). Lipid peroxidaion during myocardial reperfusion. Mol Cell Biochem 111:49-54.

Chao JC, Medeiros DM, Davidson J, and Shiry L (1994). Decreased levels of ATP synthase and cytochrome $c$ oxidase subunit peptide from hearts of copper-deficient rats are not altered by the administration of dimethyl sulfoxide. J Nutr 124:789-803.

Chen Y, Saari JT, and Kang YJ (1994). Weak antioxidant defenses make the heart a target for damage in copperdeficient rats. Free Radical Biol Med 17:529-536.

Church GM, and Gilbert W (1984). Genomic sequencing. Proc Natl Acad Sci USA 81:1991-1995.

Dhalla AK, and Singal PK (1994). Antioxidant changes in hypertrophied and failing guinea pig hearts. Am J Physiol 266:H1280-H1285.
Davis GK, and Mertz W (1987). Copper. In: Mertz W, editor. Trace elements in human and animal nutrition. New York: Academic Press, 301-364.

Eaton DL, and Cherian MG (1991). Determination of metallothionein in tissues by cadmium-hemoglobin affinity assay. Methods Enzymol 205:83-88.

Esterbauer H, and Zollner H (1989). Methods for determination of aldehydic lipid peroxidation products. Free Radical Biol Med 7:197-203.

Esterbauer H, Lang J, Zadravee S, and Slater TF (1984). Detection of malonaldehyde by high-performance liquid chromatography. Methods Enzymol 105:319-328.

Fell BF (1987). The pathology of copper deficiency in animals. In: Howell JM and Gawthorne JM, editors. Copper in animals and man. Boca Raton: CRC Press, 1-28.

Gupta M, and Singal PK (1989). Higher antioxidative capacity during a chronic stable heart hypertrophy. Circ Res 64:398406.

Hamer DH (1986). Metallothionein. Ann Rev Biochem 55: 913-951.

Hill MF, and Singal PK (1996). Antioxidant and oxidative stress changes during heart failure subsequent to myocardial infarction in rats. Am J Pathol 148:291-300.

Hill MF, and Singal PK (1997). Right and left myocardial antioxidant rsponses during heart failure subsequent to myocardial infarction. Circulation 96:2414-2420.

Jenkinson SG, Lawrence RA, Grafton WD, Gregory PE, and McKinney MA (1984). Enhanced pulmonary toxicity in copper-deficient rats exposed to hyperoxia. Fund Appl Toxicol 4:170-177.

Kagi JHR (1991). Overview of metallothionein. Methods Enzymol 205:613-626.

Kajstura J, Cheng W, Reiss K, Clark WA, Sonnenblick EH, Krajewski S, Reed JC, Olivetti G, and Anversa P (1996). Apoptotic and necrotic myocyte cell deaths are independent contributing variables of infarct size in rats. Lab Invest 74:86-107.

Kang YJ (1999). The antioxidant function of metallothionein in the heart. Proc Sci Exp Biol Med 222:263-273.

Kang YJ, Chen Y, Yu A, Voss-McCowan M, and Epstein PN (1997). Overexpression of metallothionein in the heart of transgenic mice suppresses doxorubicin cardiotoxicity. $\mathrm{J}$ Clin Invest 100:1501-1506.

Kang YJ, Li G-Q, and Saari JT (1999). Metallothionein inhibits ischemia-reperfusion injury in mouse heart. Am J Physiol 276:H993-H997.

Kang YJ, Wu H-Y, and Saari JT (2000). Alterations in hypertrophic gene expression by dietary copper restriction in mouse heart. Proc Soc Exp Biol Med 223:282-287.

Loidl-Stahlhofen A, and Spiteller G (1994). a-Hydroxyaldehydes, products of lipid peroxidation. Biochim Biophys Acta 1211:156-160.

Lorell BH (1997). Transition from hypertrophy to failure. Circulation 96:3825-3827. 
McAndrew J, Patel RP, Jo H, Cornwell T, Lincoln T, Moellering D, White CR, Matalon S, and Darley-Usmar V (1997). The interplay of nitric oxide and peroxynitrite with signal transduction pathways: Implications for disease. Seminars Perinatol 21:351-366.

Medeiros DM, Bagby GO, and McCormick R (1991). Myofibrillar, mitochondrial and valvular morphological alterations in cardiac hypertrophy among copper-deficiency rats. J Nutr 121:815-824.

Medeiros DM, Dadidson J, and Jenkins JE (1993). A unified perspective on copper deficiency and cardiomyopathy. Proc Soc Exp Biol Med 203:262-273.

Medeiros DM, and Wildman REC (1997). Newer findings on a unified perspective of copper restriction and cardiomyopathy. Proc Soc Exp Biol Med 215:299-313.

Naganuma A (1988). Specific reduction of toxic side effects of adriamycin by induction of metallothionein in mice. Jpn J Cancer Res 79:406-411.

Namikawa K, Okazaki Y, Nishida S, Kimoto S, Akai F, Tomura T, and Hashimoto S (1993). Changes in myocardial metallothionein on isoproterenol-induced myocardial injury. Yakugaku. Zasshi. 113:591-595.

Nelson SK, Huang C-J, Mathias MM, and Allen KGD (1992). Copper-marginal and copper-deficient diets decrease aortic prostacyclin production and copper-dependent superoxide dismutase activity, and increase aortic lipid peroxidation in rats. J Nutr 122:2101-2108.

Nielsen FH, Zimmerman TJ, and Shuler TR (1982). Interactions among nickel, copper and iron in rats. Liver and plasma contents of lipids and trace elements. Biol Trace Elem Res 4:125-143.

Olivetti G, Abbi R, Quaini F, Kajstura J, Cheng W, Nitahara JA, Quaini E, Loreto CD, Beltrami CA, Krajewski S, Reed JC, and Anversa $P$ (1997). Apoptosis in the failing human heart. New Engl J Med 336:1131-1141.

Prohaska JR (1990). Biochemical changes in copper deficiency. J Nutr Biochem 1:452-461.

Prohaska JR, and Heller LJ (1982). Mechanical properties of the copper-deficient rat heart. J Nutr 112:2142-2150.

Reeves PG, Nielsen FH, and Fahey GC Jr (1993). AIN-93 purified diets for laboratory rodents: Final report of the American Institute of Nutrition ad hoc writing committee on the reformulation of the AIN-76A rodent diet. J Nutr 123: 1939-1951.

Saari JT (1989). Chronic treatment with dimethyl sulfoxide protects against cardiovascular defects of copper deficiency. Proc Soc Exp Biol Med 190:121-124.
Sabbah HN, and Sharov VG (1998). Apoptosis in heart failure. Prog Cardiovas Dis 40:549-562.

Sato M, and Bremner I (1993). Oxygen free radicals and metallothionein. Free Radical Biol Med 14:325-337.

Sato M, Sasaki M, and Hojo H (1992). Tissue specific induction of metallothionein synthesis by tumor necrosis factor-alpha. Res Commun Chem Pathol Pharmacol 75:159-172.

Schwart MA, Lazo JS, Yalowich JC, Allen WP, Whitmore M, Bergonia HA, Tzeng E, Billiar TR, Robbins PD, Lancaster JR. $\mathrm{Jr}$, and Pitt BR (1995). Metallothionein protects against the cytotoxic and DNA-damaging effects of nitric oxide. Proc Natl Acad Sci USA 92:4452-4456.

Singal PK, and Kirshenbaum LA (1990). A relative deficit in antioxidant reserve may contribute in cardiac failure. Can J Cardiol 6:47-49.

Singh N, Dhalla AK, Seneviratne C, and Singal PK (1995). Oxidative stress and heart failure. Mol Cell Biochem 147:77-81.

Spitz DR, and Oberley LW (1989). An assay for superoxide dismutase activity in mammalian tissue homogenates. Anal Biochem 179:8-18.

Sunderman FW, and Nomoto S (1970). Measurement of human serum ceruloplasmin by its $\mathrm{p}$-phenylenediamine oxidase activity. Clin Chem 16:903-910.

Viestenz KE, and Klevay LM (1982). A randomized trial of copper therapy in rats with electrocardiographic abnormalities due to copper deficiency. Am J Clin Nutr 35:258-266.

Wang G-W, and Kang YJ (1999). Inhibition of doxorubicin toxicity in cultured neonatal mouse cardiomyocytes with elevated metallothionein levels. J Pharmacol Exp Ther 288: 938-944.

Wang G-W, Schuschke DA, and Kang YJ (1999). Metallothionein-overexpressing neonatal mouse cardiomyocytes are resistant to $\mathrm{H}_{2} \mathrm{O}_{2}$ toxicity. Am J Physiol 276: $\mathrm{H} 167-$ H175.

Wu C-F, Bishopric NH, and Pratt RE (1997). Atrial natriuretic peptide induces apoptosis in neonatal rat cardiac myocytes. J Biol Chem 272:14860-14866.

Wu H-Y, and Kang YJ (1998). Inhibition of buthionine sulfoximine-enhanced doxorubicin toxicity in metallothionein overexpressing transgenic mouse heart. J Pharmacol Exp Ther 287:515-520.

Yasue H, Yoshimura M, Sumida H, Kikuta K, Kugiyama K, and Jougasaki M (1994). Localization and mechanism of secretion of B-type natriuretic peptide in comparison with those of A-type natriuretic peptide in normal subjects and patients with heart failure. Circulation 90:195-203. 